\title{
Right drug, wrong dosage: insights from the PAVE-AF antithrombotic study in older patients with atrial fibrillation
}

\author{
Stylianos Tzeis ${ }^{1} \cdot$ Paraskevi Savvari $^{2} \cdot$ loannis Skiadas $^{2} \cdot$ Sotirios Patsilinakos $^{3} \cdot$ Kimon Stamatelopoulos $^{4} \cdot$ \\ Spyridon Kourouklis ${ }^{5} \cdot$ Sotirios Kyrikos ${ }^{6} \cdot$ Konstantinos Tsatiris $^{7} \cdot$ Damianos Menegas $^{2} \cdot$ George Hahalis $^{8}$. \\ George Giannakoulas ${ }^{9}$ on behalf of the PAVE-AF study group
}

Published online: 9 June 2020

(c) The Author(s) 2020

\begin{abstract}
Optimal antithrombotic treatment of older patients is usually impeded by several prevailing misconceptions. The aim of our study was to assess the type, dosage and predictors of antithrombotic therapy in older patients with non-valvular atrial fibrillation (NVAF). PAVE-AF was a prospective, cross-sectional study, including NVAF patients $\geq 80$ years from 30 participating centers. Demographic data, comorbidities and treatment patterns were documented in a single visit. Patients treated with non-vitamin K oral anticoagulants (NOACs) were further classified into three dosing categories (recommended, underdosing and overdosing). Among 1018 patients ( $85.4 \pm 4.0$ years), $88.4 \%$ received anticoagulants (AC), $8 \%$ antiplatelets (AP) and $3.6 \%$ no treatment. The primary reason for AP administration was physician concern of bleeding followed by patient denial. Patients $\geq 90$ years had two times greater probability to receive AP therapy compared to patients $<90$ years. Among patients treated with AC, one third received vitamin $\mathrm{K}$ antagonists, while two thirds received NOACs [34.6\% apixaban, 9.5\% dabigatran and 22.6\% rivaroxaban]. Independent predictors of AC prescription over AP or no treatment were low HAS-BLED score, hypertension, labile INR, permanent AF, absence of uncontrolled hypertension, prior stroke/systemic embolism, age and male gender. In total, $37 \%$ of NOAC recipients received inappropriate dosage, while the number of patients receiving recommended dosing differed significantly among NOAC subgroups $(\mathrm{p}<0.001)$. In our study, a minority of older NVAF patients received AP or no therapy for stroke prevention. Among patients treated with anticoagulants, two thirds were on NOAC treatment, though with a considerable proportion of inappropriate dosing.
\end{abstract}

Keywords Atrial fibrillation $\cdot$ Older $\cdot$ Antithrombotic $\cdot$ Dosage $\cdot$ NOAC $\cdot$ Anticoagulation

\section{Highlights}

All authors take responsibility for all aspects of the reliability and freedom from bias of the data presented and their discussed interpretation.

The members of the PAVE-AF study group are listed in acknowledgements.

Electronic supplementary material The online version of this article (https://doi.org/10.1007/s11239-020-02167-8) contains supplementary material, which is available to authorized users.

Stylianos Tzeis

stzeis@ otenet.gr

Extended author information available on the last page of the article
- Two thirds of patients $\geq 80$ years treated with oral anticoagulants receive NOACs.

- Only $63 \%$ of NOAC recipients receive appropriate dosing.

- Patients $\geq 90$ years have two times greater probability to receive antiplatelet therapy compared to patients $<90$ years.

- Low HAS-BLED, labile INR, permanent AF, male gender, and prior stroke are independent predictors of anticoagulant prescription.

- Our results raise a red flag with respect to subtherapeutic anticoagulant dosage and point out the need for continuous education on appropriate drug therapy, irrespective of patient's age. 


\section{Introduction}

Atrial fibrillation (AF) is the most common arrhythmia and a disease of aging. Population aging has substantially increased the total AF burden, but also the relative proportion of affected older patients. Therefore, overall improved care of AF patients is not feasible without focusing on the subgroup of older patients [1].

Stroke prevention with anticoagulants (AC) is a reasonable choice in older patients with AF. However, several prevailing misconceptions may impede optimal thromboprophylaxis. Advanced age per se has been reported as a prominent physician-related barrier to administration of $\mathrm{AC}$ in $\mathrm{AF}$ patients leading to underdosing [2, 3]. Another caveat is the misperception that antiplatelets (AP) are somewhat effective and definitively safer than AC rendering them an attractive "soft option" in terms of a safetydriven prescribing attitude [4].

Evaluation of real-world practice is necessary to identify pertinent treatment gaps and to improve provided healthcare services. In this context, we performed a realworld study to assess the type, dosage and predictors of antithrombotic treatment in a representative, nationwide sample of older patients over 80 years with non-valvular atrial fibrillation (NVAF). Reasons for AP use and age subgroup ( $\geq 90$ and $<90$ years) differences were also investigated.

\section{Methods}

\section{Study population}

The PAVE-AF (Pattern of Antithrombotic treatment in the Very Elderly with Atrial Fibrillation) study was a prospective, multicenter, observational study that recruited consecutive patients $\geq 80$ years with NVAF from 30 participating centers in Greece (15 hospitals and 15 private practice centers). The study protocol conformed to the ethical guidelines of the 1975 Declaration of Helsinki and was approved by the respective institutional review boards. Outpatient hospital clinics (university or state, located in either major urban centers or small towns) and private practice cardiologists were included based on their geographical distribution and in an attempt to obtain a representative sample of treating physicians. In order to maintain a nationwide, representative sample, the number of consecutively enrolled patients in each geographical region in Greece was calculated as a percentage share of the total sample size. The respective percentages were defined based on the distribution of the resident population aged $\geq 80$ years by geographical region, according to the latest revision of the 2011 Population - Housing Census in Greece as completed by Hellenic Statistical Authority (supplementary Fig. 3, supplementary Table S3) [5].

Inclusion criteria were: (a) male or female patients with confirmed NVAF (excluding moderate to severe mitral stenosis or mechanical prosthetic valves) and (b) age $\geq 80$ years. All patients signed an informed consent before their enrolment. AF had to be documented either on surface ECG or on Holter recording with a minimum duration of $30 \mathrm{~s}$. AF definition and classification were standardized based on the latest ESC guidelines [6].

Data were recorded in an electronic case report form (eCRF) and included socio-demographic information, comorbidities and type of treatment. Uncontrolled hypertension was documented in the presence of systolic blood pressure $>160 \mathrm{mmHg}$, while labile INR was defined as unstable INRs or time in therapeutic range $<60 \%$. Furthermore, the most recent blood test of creatinine levels (within 3 months) was recorded and creatinine clearance $(\mathrm{CrCl})$ was calculated based on the Cockroft-Gault equation. Patients' actual body weight was included in the respective formula. If patients received either AP only or no treatment for stroke prevention, then physicians were asked to select the reason(s) that led to this decision.

Patients treated with NOACs were classified into three dosing categories (recommended, underdosing and overdosing). Based on the approved European labels, the recommended dosing regimens were the following: Apixaban: $5 \mathrm{mg}$ twice daily (bid); dose reduction to $2.5 \mathrm{mg}$ bid if $\mathrm{CrCl}$ $15-29 \mathrm{ml} / \mathrm{min}$, or if at least two out of three criteria fulfilled: age $\geq 80$ years, body weight $\leq 60 \mathrm{~kg}$, creatinine $\geq 1.5 \mathrm{mg}$ / $\mathrm{dL}$; contraindicated if $\mathrm{CrCl}<15 \mathrm{ml} / \mathrm{min}$. Rivaroxaban: $20 \mathrm{mg}$ once daily (od); dose reduction to $15 \mathrm{mg}$ od if $\mathrm{CrCl}$ 15-49 ml/min; contraindicated if $\mathrm{CrCl}<15 \mathrm{ml} / \mathrm{min}$. Dabigatran $110 \mathrm{mg}$ or $150 \mathrm{mg}$ bid if $\mathrm{CrCl}>30 \mathrm{ml} / \mathrm{min} ; 110 \mathrm{mg}$ bid in patients $\geq 80$ years or at high risk of bleeding based on the summary of product characteristics; contraindicated if $\mathrm{CrCl}<30 \mathrm{ml} / \mathrm{min}$.

Since included patients were $\geq 80$ years, all apixabantreated patients met one criterion for dose reduction, while the recommended dose for all dabigatran-treated patients was $110 \mathrm{mg}$ bid. Patients with a contraindication to the administered NOAC due to reduced creatinine clearance levels were classified in the "overdosing" category. All data were acquired during a single visit by the investigator of each center. No follow-up visits were performed.

\section{Statistics}

We calculated that a sample size of 1020 patients would provide statistical power in the range of $88 \%$ to $98 \%$ with $5 \%$ type I error to accurately predict OAC prescription. 
Descriptive methods were used to characterize the distribution of continuous variables. For non-symmetrically distributed data, the median and interquartile range were calculated. Comparisons were performed using two sample t-test, ANOVA or Mann-Whitney test, as appropriate. For multiple comparisons a Kruskal-Wallis test was applied. Multivariate logistic regression analysis was conducted. In the case of the model selection procedures, the backward selection procedure was performed; an alpha value (level of significance) greater than $10 \%$ was used as exclusion criterion. Categorical variables were described by frequencies and related percentages per class level. Comparisons were performed using chi-square test.

\section{Results}

\section{Antiplatelet therapy or no treatment for stroke prevention}

The study population included 1018 patients [mean (SD) 85.4 (4.0) years, $54.4 \%$ females], with NVAF (paroxysmal
$32.4 \%$, persistent $8 \%$ and permanent $59.6 \%$ ). In total, 899 (88.4\%) patients received AC, $82(8 \%)$ were treated only with AP, while 37 (3.6\%) patients received no treatment. Regarding AP treatment, 37 patients received aspirin, 32 clopidogrel, 2 triflusal, while 11 patients were treated with a combination of aspirin and clopidogrel. Patient characteristics in each treatment group are presented in Table 1.

The type of administered antithrombotic treatment differed significantly among AF type groups with a higher rate of AP treatment among paroxysmal AF patients (supplementary Fig. 4). Furthermore, the type of antithrombotic treatment differed significantly among age subgroups with a lower proportion of nonagenarians receiving anticoagulants (Table 2). Nonagenarians were less likely to receive an $\mathrm{AC}$ (OR 0.472) and significantly more likely to receive AP treatment compared to patients $<90$ years (OR 2.333, $\mathrm{p}=0.001)$. Similarly, more patients aged $\geq 90$ years received no treatment versus patients $<90$ years (data not shown).

In the multivariate analysis, the following factors were significantly associated with administration of AC treatment as compared to AP or no treatment: low (0-2) HASBLED score [OR 8.62, $\mathrm{p}<0.001$ ], hypertension [OR 4.85,
Table 1 Patient characteristics per treatment group

\begin{tabular}{|c|c|c|c|c|}
\hline \multirow[t]{2}{*}{ Characteristic } & \multicolumn{3}{|c|}{ Treatment group } & \multirow[t]{2}{*}{ p-value } \\
\hline & $\begin{array}{l}\text { Antico- } \\
\text { agulant } \\
(\mathrm{n}=899)\end{array}$ & $\begin{array}{l}\text { Antiplatelet } \\
\text { only }(n=82)\end{array}$ & No treatment $(n=37)$ & \\
\hline Age (SD) & $85.1(3.8)$ & $87.5(5.0)$ & $86.1(4.2)$ & $<0.001$ \\
\hline Female gender $(\%)$ & 53.4 & 62.2 & 62.2 & 0.194 \\
\hline Weight (SD) & $74.8(12.6)$ & $73.3(11.5)$ & $68.2(13.8)$ & 0.004 \\
\hline Hypertension (\%) & 78.8 & 79.2 & 48.6 & $<0.001$ \\
\hline Diabetes (\%) & 28.6 & 36.6 & 24.4 & 0.259 \\
\hline Heart failure $^{\mathrm{a}}(\%)$ & 35.8 & 33.0 & 24.4 & 0.333 \\
\hline Prior stroke/systemic embolism (\%) & 17.2 & 19.6 & 13.6 & 0.721 \\
\hline Vascular disease $^{\mathrm{b}}(\%)$ & 25 & 29.2 & 16.2 & 0.314 \\
\hline Active smoker ${ }^{\mathrm{c}}(\%)$ & 7.6 & 6.0 & 8.2 & 0.868 \\
\hline Increased alcohol consumption ${ }^{\mathrm{d}}(\%)$ & 4.4 & 6.0 & 0.0 & 0.317 \\
\hline Uncontrolled hypertension ${ }^{\mathrm{e}}(\%)$ & 8.4 & 17.0 & 8.2 & 0.03 \\
\hline Pulmonary disease $(\%)$ & 21.0 & 18.2 & 24.4 & 0.742 \\
\hline Prior bleeding or high bleeding risk (\%) & 17.2 & 26.8 & 48.6 & $<0.001$ \\
\hline Coronary heart disease (\%) & 24.4 & 28.0 & 10.8 & 0.117 \\
\hline Angioplasty/stenting within prior year (\%) & 2.6 & 3.6 & 0.0 & 0.504 \\
\hline CHADSVASc score (SD) & $4.6(1.4)$ & $4.8(1.2)$ & $4.0(1.4)$ & 0.009 \\
\hline HAS-BLED score (SD) & $2.4(0.9)$ & $3.0(1.0)$ & $2.4(0.9)$ & $<0.001$ \\
\hline
\end{tabular}

$S D$ standard deviation

${ }^{a}$ Heart failure: signs/symptoms of heart failure and/or left ventricular ejection fraction $<40 \%$

${ }^{b}$ Vascular disease: history of myocardial infarction or peripheral arterial disease or presence of atherosclerotic plaque in the aorta

${ }^{\mathrm{c}}$ Active smoker: more than two cigarettes daily for at least 2 years

${ }^{\mathrm{d}}$ Increased alcohol consumption: more than 8 drinks per week

${ }^{\mathrm{e}}$ Uncontrolled hypertension: resting systolic blood pressure $>160 / 90 \mathrm{mmHg}$ 
Table 2 Type of antithrombotic treatment administered in different age subgroups

\begin{tabular}{lllll}
\hline Antithrombotic treatment & Age subgroup & & p value \\
\cline { 2 - 4 } & $80-84$ years $(\mathrm{n}=496)$ & $85-89$ years $(\mathrm{n}=357)$ & $\geq 90$ years $(\mathrm{n}=165)$ & \\
\hline Anticoagulants & $452(91.2 \%)$ & $314(88 \%)$ & $133(80.6 \%)$ & 0.005 \\
Antiplatelet monotherapy & $28(5.6 \%)$ & $30(8.4 \%)$ & $24(14.6 \%)$ & \\
No treatment & $16(3.2 \%)$ & $13(3.6 \%)$ & $8(4.8 \%)$ & \\
\hline
\end{tabular}

Fig. 1 Multivariate predictors of treatment with anticoagulants versus antiplatelets or no treatment

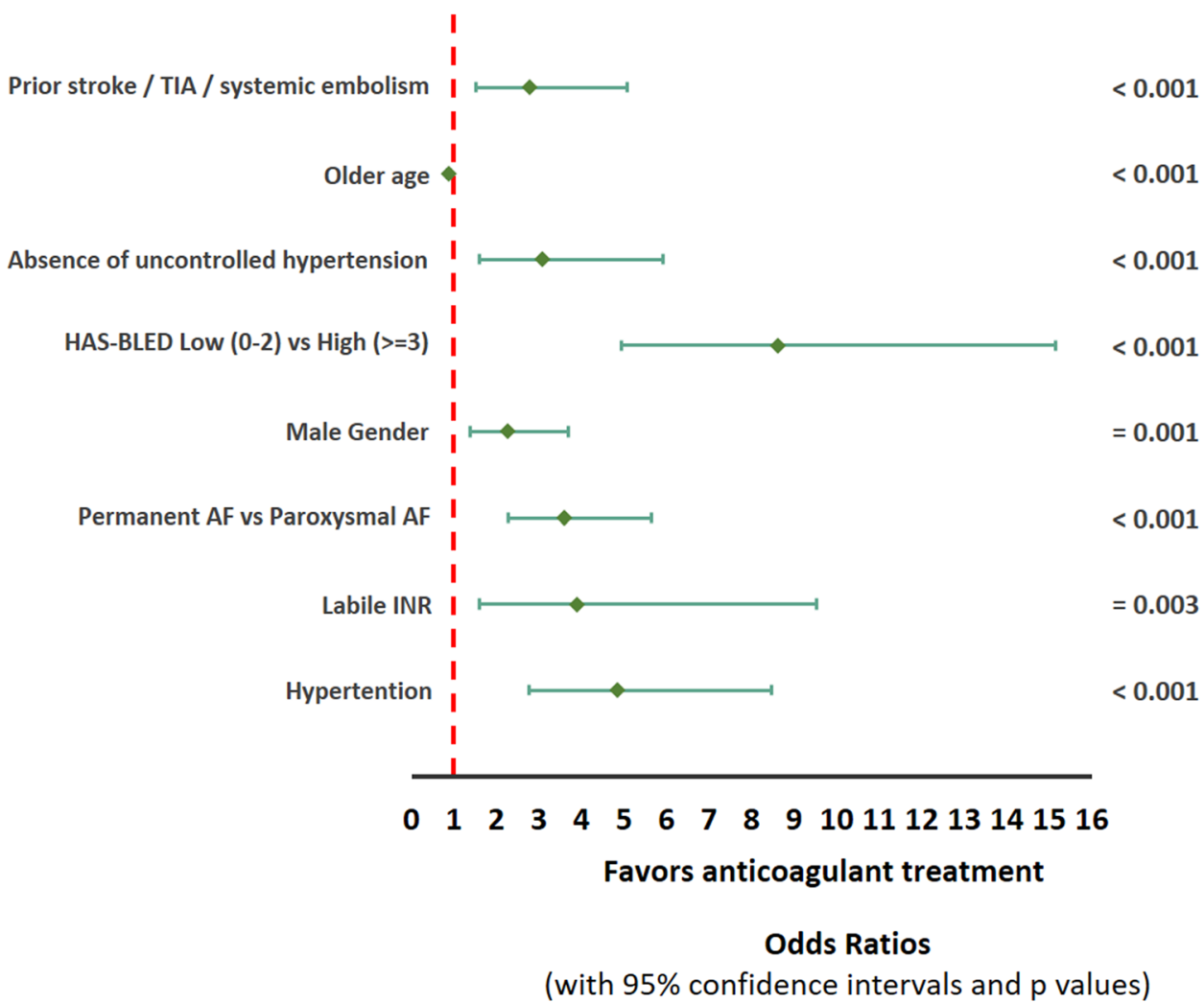

$\mathrm{p}<0.001$ ], labile INR [OR 3.89, $\mathrm{p}=0.003$ ] permanent AF [OR 3.60, $\mathrm{p}<0.001]$, absence of uncontrolled hypertension [OR 3.07, p < 0.001], prior stroke/systemic embolism [OR $2.78, \mathrm{p}<0.001$ ], age (continuous variable - per year of age) [OR 0.87, p <0.001] and male gender [OR 2.26, $\mathrm{p}=0.001]$ (Fig. 1).

\section{Reasons for antiplatelet monotherapy or no treatment}

Physicians reported that the following reason(s) led to their decision to administer either AP only or no treatment for stroke prevention: anticipated high bleeding risk in 57 $(31.2 \%)$ patients, patient denial in $56(30.6 \%)$, inability of regular INR monitoring in 41 (22.4\%), adverse event on prior anticoagulant treatment in $23(12.6 \%)$, inability to maintain INR within therapeutic range in $22(12 \%)$, high treatment cost in $10(5.4 \%)$ and other reasons in $20(11 \%)$ patients.

\section{Type and dosage of anticoagulant treatment}

Among 899 patients treated with anticoagulants, 21 received parenteral and 878 oral antithrombotic treatment. In the latter subgroup, 293 patients were treated with vitamin $\mathrm{K}$ antagonists $(33.4 \%)$, while $585(66.6 \%)$ received NOACs [304 (34.6\%) apixaban, $83(9.5 \%)$ dabigatran and 198 (22.6\%) rivaroxaban]. A significantly lower percentage of patients $\geq 90$ years were treated with NOACs as compared to patients aged $80-89$ years ( $48.4 \%$ vs $59.2 \%$, respectively, $\mathrm{p}<0.05)$. The reduced NOAC doses were administered in $71.8 \%$ of apixaban-treated patients $(2.5 \mathrm{mg}$ bid), in $82 \%$ of dabigatran-treated patients (110 mg bid) and in $63.2 \%$ of rivaroxaban-treated patients (15 mg od). In total, $63.2 \%$ of patients received NOAC dosing consistent with European label recommendations, $29.7 \%$ received a lower dose, while $7.1 \%$ were overdosed (supplementary Table S4). The percentage of patients receiving recommended, over- or 


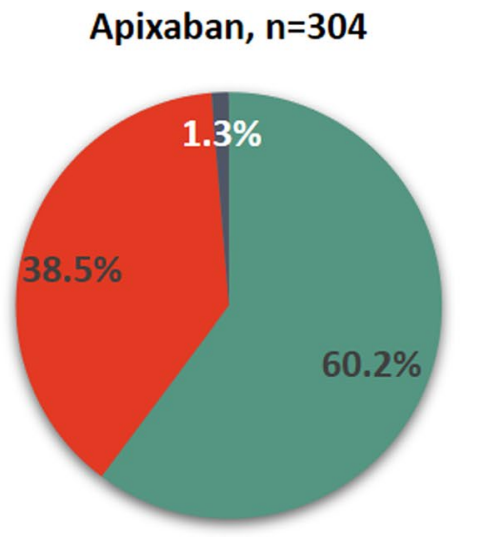

Recommended
Dabigatran, $n=83$

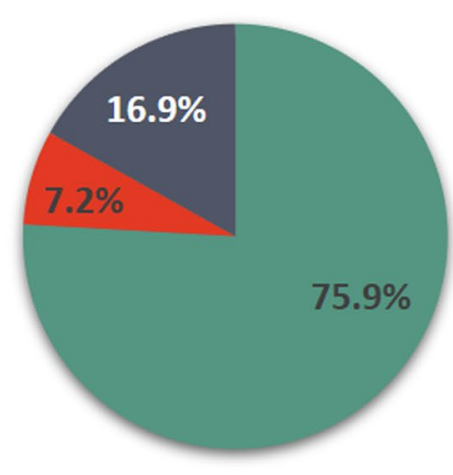

Underdosing
Rivaroxaban, $\mathrm{n}=198$

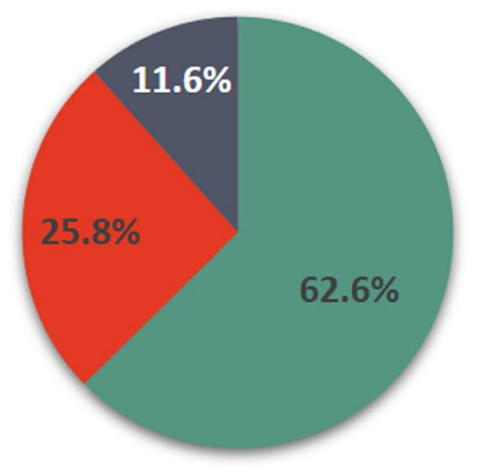

Overdosing

Fig. 2 Percentage of NOAC recipients receiving recommended dosing, overdosing or underdosing

under- dosing differed significantly among the three NOAC groups (p<0.001), (Fig. 2).

\section{Discussion}

The main finding of this prospective, nationwide, representative, real-world study is that $11.6 \%$ of patients $>80$ years with NVAF receive antiplatelet monotherapy or no treatment for stroke prevention. Among patients treated with oral anticoagulants, two thirds are under NOAC treatment. However, only $63.2 \%$ of NOAC recipients receive appropriate dosing based on European label recommendations.

\section{Antiplatelet treatment for stroke prevention}

The latest ESC guidelines do not recommend antiplatelet monotherapy for stroke prevention in AF patients [6]. However, there has always been a considerable time lag before issued recommendations are incorporated into everyday practice. Several real-world surveys have reported the persistence of antiplatelet use for stroke prevention in AF patients [7-10]. However, most registries do not provide detailed data regarding prescription patterns in older patients. Furthermore, reported findings pertain to a transient period following approval and initial market penetration of different NOACs. This dynamic state may have prevented the capture of a steady and representative prescription behavior of physicians for stroke prevention. Our study enrolled patients several years post launch of NOACs in the Greek market, where the existing pricing and reimbursement policy allows patient access to this pharmacological treatment, thus reducing potential confounding effect of drug availability and affordability. In our study we found that $8 \%$ of real-world AF patients over 80 years are treated only with antiplatelets for stroke prevention, while only $3.6 \%$ receive no treatment at all. The most frequently reported reasons why an anticoagulant was not administered included anticipated high bleeding risk, patient denial and inability of regular INR monitoring, while treatment cost influenced therapeutic choices in a minority of cases. Our study also demonstrated several independent predictors associated with administration of antiplatelet monotherapy or no treatment in older patients over 80 years with AF, including increased bleeding risk (high HAS-BLED score), older age, uncontrolled hypertension, female gender and history of paroxysmal AF.

\section{NOAC type and dosing in older AF patients}

NOACs have evidence-based credentials for stroke prevention in patients with NVAF [11]. Their advent has signaled a shift in the prescribing pattern of therapies for stroke prevention. A Danish nationwide registry showed a significant decrease in the usage of vitamin $\mathrm{K}$ antagonists from 92 to $24 \%$, with a counterbalancing increase in NOAC use [12]. Real world data evaluating the use of NOACs in older AF patients are scarce. In our study, two thirds of patients treated with oral anticoagulants received NOACs, thus supporting increased utilization of NOACs over vitamin $\mathrm{K}$ antagonists.

Apart from the standard doses, reduced NOAC dosing schemes have been approved for predefined patient subgroups with certain baseline characteristics [11]. The administration of reduced NOAC dosing in patients not meeting the recommended criteria worsens patient outcome and significantly increases the risk of adverse events [13]. NOAC overdosing was reported to increase all-cause mortality, while underdosing increased cardiovascular hospitalizations as compared to recommended doses [13]. The reported incidence of inappropriate NOAC dosing varies. Steinberg et al. have reported that only $13 \%$ of AF patients treated 
with NOACs received off-label dosing [13]. This percentage is considerably lower than the respective in our study. We found that $29.7 \%$ of patients received a lower dose, while $7.1 \%$ were overdosed. Several factors may explain this discrepancy. The authors have commented that the reported results may underestimate the magnitude of incorrect dose selection in broader AF populations, due to the potential confounding effect of participation in an AF registry [13]. The ORBIT-AF II registry evaluated the US community practice and ascertainment of appropriate NOAC dosing was based on the FDA-approved package inserts that differ from the respective European labels. Thus, the use of different classification criteria limits the extrapolation of relevant findings in the European care setting. Furthermore, the ORBIT-AF II analysis included a general AF patient population, with a mean age of 70 years, while our study specifically focused in the older patient subpopulation over 80 years. The popularity of a "softer" anticoagulant prescribing practice among AF patients over 80 years due to concerns on frailty, bleeding and falling, may partly explain the increased rate of underdosing [14]. In line with our results, Barra et al., in a retrospective chart review of 224 patients with a mean age of 77.7 years who received a reduced NOAC dose, found that only $43.3 \%$ of patients satisfied the dose adjustment criteria recommended by the manufacturer labeling [15].

Our findings reflect physicians' willingness to avoid bleeding complications in the context of a safety-driven decision-making in anticoagulant type and dosage. Interestingly, there is a shift in the adopted "soft" thromboprotective options from antiplatelet monotherapy to inappropriately low doses of NOACs. However, inappropriate NOAC dosing in the older AF patients still deprives them of optimal thromboprotection. Intensive educational campaigns are needed to further familiarize prescribing physicians with the indicated NOAC dosing schemes and to educate them on the unfavorable prognostic impact of inappropriate NOAC dosing.

\section{Strengths and limitations}

Several methodological strengths of our study should be commented. PAVE-AF is a prospective non-interventional study which is considered to provide evidence of "higher quality" than retrospective claims analyses. The latter is a common source of real-world evidence, but prone to bias due to void selection of diagnosis coding, missing data and lenient case definition. In our study, AF was confirmed either by surface ECG or on Holter monitoring with a duration of at least $30 \mathrm{~s}$. Furthermore, we used minimal exclusion criteria to increase the external validity of our results. The study population had a representative geographical distribution to reduce the likelihood of systematic enrolment bias and to increase the generalizability of our findings. The number of patients enrolled in each geographical region was proportional to the reference population ( $\geq 80$ years), based on the latest national population census. The participating centers have been selected on the basis of including different care setting for AF patients including both physician offices, as well as different types of hospitals. In addition, consecutive sampling was employed with a predefined target size to enhance the representativeness of the reference population and all eCRFs were validated through source data verification processes. However, strict implementation of consecutive patient enrolment cannot be confirmed, since use of screening logs was not mandated by the study protocol; thus, selection bias cannot be excluded. Another limitation of the study is the lack of follow-up data that do not allow the evaluation of patient outcome.

\section{Clinical implication}

The results of our real-world study contribute to the identification of antithrombotic treatment pattern in older NVAF patients, a population with limited data in the literature. Based on our findings, older patients with NVAF are mostly treated with anticoagulants and preferably with a NOAC. However, nonagenarians are twice likely to receive an AP or no treatment mainly due to physician's fear of bleeding. Most importantly, more than one third of our population receive inappropriate dosage. Although our results are encouraging and partly imply adherence to current treatment guidelines, they raise a red flag with respect to inappropriate dosage, pointing out the need for continuous education on appropriate drug therapy irrespective of patient's age.

\section{Conclusion}

In the real world, a minority of older patients $>80$ years with non-valvular AF receives antiplatelet monotherapy or no treatment for stroke prevention. Among patients treated with oral anticoagulants, two thirds are on NOAC treatment, but inappropriate dosing remains a significant barrier preventing optimal patient thromboprophylaxis.

Acknowledgements The authors would like to thank Dr. Periklis Giovas for his substantial contribution to the conception/design of the study and Dr. Dimitrios Chatzis for his contribution to data analysis.

PAVE-AF study group investigators: Dimitrios Chryssos, Georgios Diakakis, Konstantinos Gkouias, Krystallenia Kafkala, Maria Kantziou, Athanasios Kapetanopoulos, Petros Kikas, Petros Kirytopoulos, Dimitrios Korres, Efstathios Koulouris, Konstantinos Kyratlidis, Aggeliki Laina, Stylianos Lampropoulos, Georgios Lymperopoulos, Dimitrios Makrygiannis, Athanasios Maragiannis, Theodoros Michailidis, Irini Mpourni, Efthymia Nasothymiou, Christoforos Olympios, Dimitrios Papadogiannis, Eleni Paphianou, Neofytos Papoulidis, Athanasios 
Protogerou, Dionysia Ralli, Panagiotis Rigopoulos, Ilias Sihlimiris, Stavros Spanodimos, Christos Stathopoulos, Maria Toumpourleka, Grigorios Tsigkas, Dimitrios Tziakas, Thomas Tzimas, Nikiforos Vrettos, Tsilla Zafiriou, Aristides Zafiris, Georgios Zonios.

Author contributions ST had substantial contributions to conception and design of the study, as well as the acquisition, the analysis and interpretation of data. He has also written the manuscript. PS had substantial contribution to design, interpretation of data and revision of the manuscript for important intellectual content. IS had substantial contribution to interpretation of data and revision of the article content. SP, KS, SK, SK, KT, GH and GG had substantial contributions to the acquisition of the data and final approval of the version to be published. DM had critically revised the manuscript for important intellectual content and approved the final version to be published. The PAVE-AF study group investigators (listed in acknowlegdements) had substantial contribution to acquisition of the data.

Funding This work was funded by Pfizer Hellas SA. The sponsor has not interfered in the acquisition and the analysis of the data, as well as the drafting of the manuscript.

\section{Compliance with ethical standards}

Conflict of interest Paraskevi Savvari, John Skiadas and Damianos Menegas are currently Pfizer employees. The rest of the authors have no conflicts.

Ethical approval The study protocol conformed to the ethical guidelines of the 1975 Declaration of Helsinki and was approved by the respective institutional review boards.

Informed consent All listed authors have approved the manuscript before submission. All listed authors consent to publication.

Open Access This article is licensed under a Creative Commons Attribution 4.0 International License, which permits use, sharing, adaptation, distribution and reproduction in any medium or format, as long as you give appropriate credit to the original author(s) and the source, provide a link to the Creative Commons licence, and indicate if changes were made. The images or other third party material in this article are included in the article's Creative Commons licence, unless indicated otherwise in a credit line to the material. If material is not included in the article's Creative Commons licence and your intended use is not permitted by statutory regulation or exceeds the permitted use, you will need to obtain permission directly from the copyright holder. To view a copy of this licence, visit http://creativecommons.org/licenses/by/4.0/.

\section{References}

1. Krijthe BP, Kunst A, Benjamin EJ, Lip GY, Franco OH, Hofman A et al (2013) Projections on the number of individuals with atrial fibrillation in the European Union, from 2000 to 2060. Eur Heart J 34:2746-2751
2. Lip GY, Andreotti F, Fauchier L, Huber K, Hylek E, Knight E et al (2011) Bleeding risk assessment and management in atrial fibrillation patients: a position document from the European Heart Rhythm Association, endorsed by the European Society of Cardiology Working Group on thrombosis. Europace 13:723-746

3. Pugh D, Pugh J, Mead GE (2011) Attitudes of physicians regarding anticoagulation for atrial fibrillation: a systematic review. Age Ageing 40:675-683

4. Ben Freedman S, Gersh BJ, Lip GY (2015) Misperceptions of aspirin efficacy and safety may perpetuate anticoagulant underutilization in atrial fibrillation. Eur Heart J 36:653-656

5. Demographic and social characteristics of the resident population of Greece according to the 2011 Population-Housing census revision of 20/3/2014. Available at: http://www.statistics.gr/en/stati stics/-/publication/SAM03/2011

6. Kirchhof P, Benussi S, Kotecha D, Ahlsson A, Atar D, Casadei $B$ et al (2016) 2016 ESC guidelines for the management of atrial fibrillation developed in collaboration with EACTS. Europace 18:1609-1678

7. Proietti M, Laroche C, Opolski G, Maggiono AP, Boriani G, Lip GYH, Gen Pilot Investigators AF (2017) Real-world' atrial fibrillation management in Europe: observations from the 2-year follow-up of the EURObservational research programme-atrial fibrillation general registry Pilot phase. Europace 19:722-733

8. Huisman MV, Ma CS, Diener HC, Dubner SJ, Halperin JL, Rothman KJ et al (2016) Antithrombotic therapy use in patients with atrial fibrillation before the era of non-vitamin $\mathrm{K}$ antagonist oral anticoagulants: the Global Registry on Long-Term Oral Antithrombotic Treatment in Patients with Atrial Fibrillation (GLORIA-AF) phase I cohort. Europace 18:1308-1318

9. Huisman MV, Rothman KJ, Paquette M, Teutsch C, Diener HC, Dubner SJ et al (2017) The changing landscape for stroke prevention in AF: findings from the GLORIA-AF registry phase 2. J Am Coll Cardiol 69:777-785

10. Camm AJ, Accetta G, Ambrosio G, Atar D, Bassand JP, Berge E et al (2017) Evolving antithrombotic treatment patterns for patients with newly diagnosed atrial fibrillation. Heart 103:307-314

11. Steffel J, Verhamme P, Potpara TS, Albaladejo P, Antz M, Desteghe L et al (2018) The 2018 European Heart Rhythm Association Practical Guide on the use of non-vitamin K antagonist oral anticoagulants in patients with atrial fibrillation: executive summary. Europace 20:1231-1242

12. Staerk L, Fosbøl EL, Gadsbøll K, Sindet-Pedersen C, Pallisgaard JL, Lamberts M et al (2016) Non-vitamin K antagonist oral anticoagulation usage according to age among patients with atrial fibrillation: temporal trends 2011-2015 in Denmark. Sci Rep 6:31477

13. Steinberg BA, Shrader P, Thomas L, Ansell J, Fonarow GC, Gersh BJ et al (2016) Off-label dosing of non-vitamin K antagonist oral anticoagulants and adverse outcomes: the ORBIT-AF II registry. J Am Coll Cardiol 68:2597-2604

14. Scowcroft AC, Lee S, Mant J (2013) Thromboprophylaxis of elderly patients with AF in the UK: an analysis using the General Practice Research Database (GPRD) 2000-2009. Heart 99:127-132

15. Barra ME, Fanikos J, Connors JM, Sylvester KW, Piazza G, Goldhaber SZ (2016) Evaluation of dose-reduced direct oral anticoagulant therapy. Am J Med 129:1198-1204 


\section{Affiliations}

Stylianos Tzeis ${ }^{1} \cdot$ Paraskevi Savvari $^{2} \cdot$ loannis Skiadas $^{2} \cdot$ Sotirios Patsilinakos $^{3} \cdot$ Kimon Stamatelopoulos $^{4}$. Spyridon Kourouklis ${ }^{5} \cdot$ Sotirios Kyrikos $^{6} \cdot$ Konstantinos Tsatiris $^{7} \cdot$ Damianos Menegas $^{2} \cdot$ George Hahalis $^{8}$. George Giannakoulas ${ }^{9}$ on behalf of the PAVE-AF study group

1 Cardiology Department, Mitera General Hospital, Hygeia Group, 6, Erithrou Stavrou, Maroussi, 15123 Athens, Greece

2 Medical Department of Internal Medicine, Pfizer Hellas, Athens, Greece

3 Cardiology Department, General Hospital "Agia Olga", Athens, Greece

4 Therapeutic Department, General Hospital "Alexandra", Athens, Greece

5 5th Cardiology Department, Hygeia Hospital, Athens, Greece
6 Cardiology Department, General Hospital of Eastern Achaia-Aegio, Aegio, Greece

7 Cardiology Department, General Hospital Karditsa, Karditsa, Greece

8 Cardiology Department, University General Hospital Patras, Patras, Greece

9 Cardiology Department, University General Hospital "AHEPA", Thessaloniki, Greece 